\title{
Repulsive Particle Method Considering Escaping Local Minima in Unknown Environment
}

\author{
Daiki Koike, Kenji Uchiyama \\ Nihon University \\ Funabashi, Chiba 274-8501, Japan \\ csda13039@g.nihon-u.ac.jp; uchiyama@aero.cst.nihon-u.ac.jp
}

\begin{abstract}
This paper describes an efficient guidance method for a space rover in an unknown and GPS-denied environment. In an unknown planet, it is effective for an exploration to use a method called FastSLAM that performs simultaneously localization and mapping using combination of a particle filter and extended Kalman filter. On the other hand, a guidance low based on the potential function method has been used because it does not require path planning in advance and is enable to respond to the sudden topographic change. However, using these methods, local minima problem in the potential field is frequently occurred when designing repulsive potential functions so as to avoid landmarks. The great advantage of the proposed method is that it is simple to escape local minima by using a repulsive particle, which is designed by using particles in a particle filter. Experiments are performed to confirm the effectiveness of the proposed method.
\end{abstract}

Keywords: FastSLAM; Potential Function Method; Repulsive Particle; Space Rover; Local Minima

\section{Introduction}

It is practical and effective to perform an exploration by using a space rover in a planet where manned exploration is impossible. Conventional space rover identifies its position and designs a path to a destination based on terrain images from a satellite orbiting a planet. However, a space rover is impossible to identify its position and design a path to a destination in case a space rover explores a planet on which an artificial satellite does not exist, or mutual communication with an orbiting satellite is interrupted on account of an unexpected accident. Furthermore, the conventional method does not respond quickly to a sudden change in terrain because a space rover normally designs a path in advance.

For such a situation, the method using SLAM and potential function method is considered as one of the effective methods. SLAM is the method by which a space rover performs localization and mapping simultaneously with sensors mounted on a space rover, and the potential function method is the way of steering to a destination and avoiding obstacles without a designed path in advance by generating an artificial potential field, i.e., a space rover can perform autonomous exploration in an unknown and GPS-denied environment with these ways. However, a space rover sometimes falls into local minima generated by a dense area of obstacles. In such a case, an exploration of a space rover will be interrupted, and continuation of a mission will be impossible. Adaptive Temperature Parallel Simulated Annealing (ATPSA), which is one of the global optimum solution search method, has been applied to a space rover to solve this problem $[1,6,7]$. This method requires iterative calculation in order to search the solution. As a result, it is difficult to implement ATPSA in actual equipment because it needs a great deal of time to escape local minima. In addition, there is also method combining genetic algorithm and the potential function method [8]. The method has also the problem with respect to the calculation time to solve the problem. Moreover, it is also important that a space rover detects accurately local minima to escape the position effectively. Gradient of velocity of a space rover was utilized to detect local minima in the method. However, there is a possibility to misrecognize a local minimum by using the decision method.

We propose the method that combines the detecting local minima with use of particles in FastSLAM and the escaping local minima with potential function method. The number of resampling, which is one of processes in FastSLAM, varies with topography when a space rover maps an area. This variation is applied to the detection of local minima, and a space rover escapes local minima without iterative calculation by generating a repulsive potential on 
particles in FastSLAM. The proposed method has advantage in terms of reduction of calculation load in comparison with ATPSA. Moreover, the number of times of resampling is possible to detect local minima more accurately than the conventional method. We confirm the effectiveness of the proposed method through the experiments.

\section{Guidance of Space Rover \\ 2.1. Equation of Motion}

Fig. 1 shows definition of variable and a coordinate system related to the kinematics of a space rover. Then, the observation equation and the discrete-time state equation of a space rover are expressed by the following equation. It is assumed that the motion of a space rover is restricted in $x y$ plane.

$$
\begin{gathered}
\mathbf{x}_{R}(k+1)=\mathbf{f}\left(\mathbf{x}_{R}(k), \omega(k), V(k)\right)+\mathbf{e}_{\mathbf{w}}(k) \\
\mathbf{f}\left(\mathbf{x}_{R}(k), \omega(k), V(k)\right)=\left[\begin{array}{c}
x_{k}(k)+T V(k) \cos \theta_{R}(k) \\
y_{\mathrm{R}}(k)+T V(k) \sin \theta_{R}(k) \\
\theta_{\mathrm{R}}(k)+T \omega(k)
\end{array}\right] \\
\mathbf{z}(k)=\mathbf{h}\left(\mathbf{x}_{l / R}(k)\right)+\mathbf{e}_{\mathbf{v}}(k) \\
\mathbf{h}\left(\mathbf{x}_{l / R}(k)\right)=\left[\begin{array}{c}
r_{l / R}(k) \\
\varphi_{l / R}(k)
\end{array}\right]=\left[\begin{array}{c}
\sqrt{x_{l / R}(k)^{2}+y_{l / R}(k)^{2}} \\
\tan ^{-1}\left\{y_{l / R}(k) / x_{l / R}(k)\right\}-\theta_{R}(k)
\end{array}\right]
\end{gathered}
$$

where $\mathbf{f}\left(\mathbf{x}_{R}(k), \omega(k), V(k)\right)$ denotes the nonlinear function with state variables of a space rover, $\mathbf{h}\left(\mathbf{x}_{l / R}(k)\right)$ the nonlinear function of observation, $\mathrm{x}_{R}(k) \in \mathrm{R}^{3}$ the state vector of a space rover, $x_{R}(k)$ the variable along $x$ axis, $y_{R}(k)$ the variable along $y$ axis, $\theta_{R}(k)$ the attitude angle, $\mathbf{x}_{l} \in \mathrm{R}^{2}$ the state vector of the $l$-th landmark, $V(k)$ the translational velocity, $\omega(k)$ the angular velocity, $\mathbf{x}_{l / R}(k)=\mathbf{x}_{l}-\mathbf{x}_{R}(k)$ the relative position vector between a space rover and $l$-th landmark, $x_{l / R}(k)=x_{l}-x_{R}(k), y_{l / R}(k)=y_{l}-y_{R}(k)$ the relative distance calculated from position of a space rover and $l$-th landmark in $x$ and $y$ direction, $r_{l / R}$ the relative distance between a space rover and $l$-th landmark, $\varphi_{l / R}$ the relative angle between a space rover and $l$-th landmark, $T$ the sampling period, $\mathbf{e}_{\mathbf{w}}(k) \in \mathrm{R}^{3}$ the process noise with covariance matrix $\mathbf{R}$, $\mathbf{e}_{\mathbf{v}}(k) \in \mathrm{R}^{3}$ the observation noise with covariance matrix $\mathbf{Q}$. The equation of motion considers a slip of wheels of the space rover sensor noise.

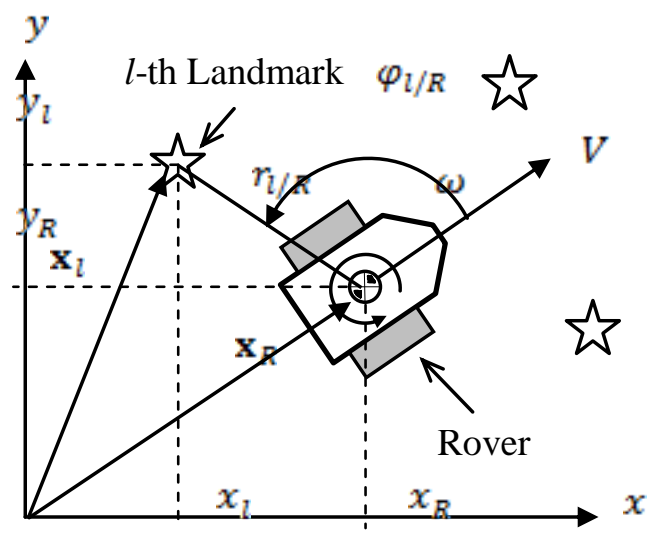

Fig. 1: Definition of state variables of space.

\subsection{Potential Function Method}


The potential function method is the way of steering a space rover to the destination and avoiding landmarks by generating an artificial potential field in an exploration area. In this method, the steering potential field is applied to guide a space rover to the destination, and the repulsive potential field is designed to avoid the observed landmarks. The potential function $U\left(\mathbf{x}_{d / R}\right)$ consists of the sum of the steering potential function $U^{S}\left(\mathbf{x}_{d / R}\right)$ and the repulsive potential function $U^{R}\left(\mathbf{x}_{l / R}\right)$ as shown in the following equation. Figs. 2 and 3 show an integrated potential field and its velocity field $[1,5]$.

$$
\begin{array}{r}
U^{S}\left(\mathbf{x}_{d / R}\right)=C_{s} \sqrt{\left|\mathbf{x}_{d / R}\right|^{2}+L_{s}} \\
U^{R}\left(\mathbf{x}_{l / R}\right)=C_{r} \sum_{l} \exp \left(-\frac{\left|\mathbf{x}_{l / R}\right|}{L_{r}}\right)
\end{array}
$$

where $C_{s}$ expresses the magnitude of the gradient of the steering potential field, $L_{s}$ the gradient variation in the vicinity of the equilibrium point, $C_{r}$ the magnitude of the gradient of the repulsive potential, $L_{r}$ the influence area of the repulsive potential. $\mathbf{x}_{d / R}=\mathbf{x}_{d}-\mathbf{x}_{R}$ represents the relative position vector between a space rover and the destination. The command to steer a space rover is calculated from the velocity field in $x$ and $y$ direction. They are obtained as follows:

$$
\begin{aligned}
& V_{x}=-\frac{\partial U^{S}\left(\mathbf{x}_{d / R}\right)}{\partial x}-\frac{\partial U^{R}\left(\mathbf{x}_{l / R}\right)}{\partial x} \\
& V_{y}=-\frac{\partial U^{S}\left(\mathbf{x}_{d / R}\right)}{\partial y}-\frac{\partial U^{R}\left(\mathbf{x}_{l / R}\right)}{\partial y}
\end{aligned}
$$

By using these equations, the velocity command $V_{d}$ and the heading angle command $\theta_{d}$ are derived as the following equation.

$$
\begin{aligned}
& V_{d}=\sqrt{V_{x}^{2}+V_{y}^{2}} \\
& \theta_{d}=\tan ^{-1}\left(\frac{V_{y}}{V_{x}}\right)
\end{aligned}
$$

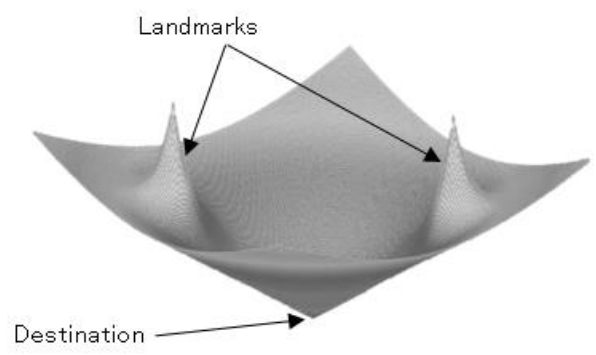

Fig. 2: Integrated potential function. 


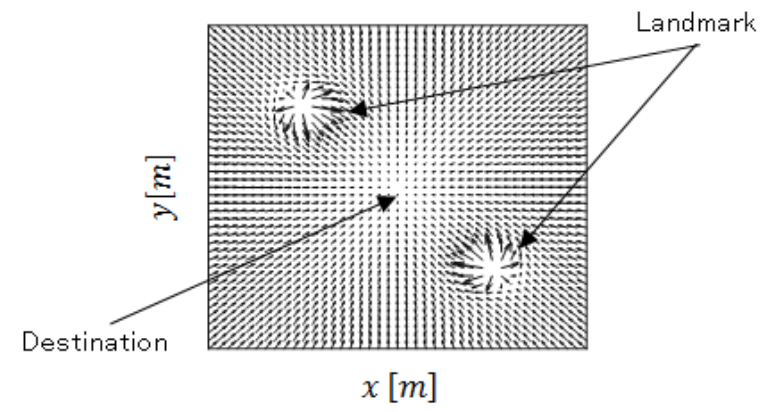

Fig. 3: Integrated velocity field.

\section{Method of Escaping Local Minima}

\subsection{FastSLAM}

SLAM is the way by which a space rover enables to map an unknown area and to localize on the basis of observation obtained from sensors mounted on a space rover. [1-4]

In this study, we apply FastSLAM to the space rover, and carry out an exploration mission. FastSLAM uses the particle filter for localization of a space rover and the extended Kalman filter for mapping of an unknown environment.

FastSLAM separates the mapping from the localization to calculate the probability as the following equation [2-4].

$P(x, s \mid z, u, c)=P(x \mid z, u, c) \prod_{l=1}^{n} P(s \mid x, z, u, c)$

where $x$ denotes the state variable of a space rover, $s$ the map of an environment, $z$ the observation obtained from its sensors, $u$ the input, $c$ the feature quantity of observed landmarks, $l$ the $l$-th $(1 \leq l \leq n)$ landmark, $n$ the number of landmarks. A space rover is able to use the particle filter to estimate its trajectory and the low dimensional extended Kalman filter to construct a map with the property of (11).

The state variable of $p$-th $(1 \leq p \leq m)$ particle is defined as the following equation in FastSLAM.

$[\mathbf{Y}(k)]_{p}=\left\langle[\mathbf{x}(k)]_{p},\left[\boldsymbol{\mu}_{l}(k)\right]_{p},\left[\mathbf{\Sigma}_{l}(k)\right]_{p}, \ldots,\left[\boldsymbol{\mu}_{n}(k)\right]_{p},\left[\mathbf{\Sigma}_{n}(k)\right]_{p}\right\rangle$

Here $[\mathbf{Y}(k)]_{p}$ expresses $p$-th particle set at time $k, \mathbf{x}(k)$ the state variable of a space rover to estimate its trajectory, $\boldsymbol{\mu}_{l}(k)$ and $\mathbf{\Sigma}_{l}(k)$ the mean and the covariance matrix of estimated $l$-th landmark, respectively. The posterior probability in FastSLAM is expressed by a total of $m$ particles. The new particle set $\mathbf{Y}(k)$ is generated from a particle set $\mathbf{Y}(k-1)$ at time $(k-1)$ when the posterior probability at time $k$ is filtered from the one at time $(k-1)$. The generated particle set $\mathbf{Y}(k)$ reflects the input $u$ and the observation $z$. The update is performed in the following steps.

\subsubsection{Prediction}

In FastSLAM, the input $u$ is employed to sample the state variable $\mathbf{x}(k)$ of a space rover from the particle set $\mathbf{Y}(k-1)$, i.e., the attitude of $p$-th particle at time $k$ is sampled based on the following equation.

$[\mathbf{x}(k)]_{p}=P\left([\mathbf{x}(k)]_{p} \mid[\mathbf{x}(k-1)]_{p}, u\right)$

Here, $[\mathbf{x}(k-1)]_{p}$ shows the estimated attitude of a space rover at time $(k-1)$, and the obtained $[\mathbf{x}(k)]_{p}$ is added to the assumed particle set with the previous trajectory $[\mathbf{x}(1 ; k-1)]_{p}$.

\subsubsection{Measurement Update}


The posterior probability of landmark expressed the mean $\left[\boldsymbol{\mu}_{l}(k-1)\right]_{p}$ and covariance $\left[\mathbf{\Sigma}_{l}(k-1)\right]_{p}$ is updated in FastSLAM. The new mean $\left[\boldsymbol{\mu}_{l}(k)\right]_{p}$ and covariance $\left[\boldsymbol{\Sigma}_{l}(k)\right]_{p}$ at time $k$ is calculated with EKF measurement update. $\left[\mu_{l}(k)\right]_{p}=\left[\mu_{l}(k-1)\right]_{p}+[\mathbf{K}(k)]_{p}\left(\mathbf{z}(k)-[\hat{\mathbf{z}}(k)]_{p}\right)$

$\left[\mathbf{\Sigma}_{l}(k)\right]_{p}=\left(\mathbf{I}-[\mathbf{K}(k)]_{p}[\mathbf{H}(k)]_{p}\right)\left[\mathbf{\Sigma}_{l}(k-1)\right]_{p}$

In which, $[\mathbf{K}(k)]_{p}$ is the Kalman gain that is given by

$[\mathbf{K}(k)]_{p}=\left[\mathbf{\Sigma}_{l}(k-1)\right]_{p}[\mathbf{H}(k)]_{p}\left([\mathbf{H}(k)]_{p}\left[\mathbf{\Sigma}_{l}(k-1)\right]_{p}[\mathbf{H}(k)]_{p}{ }^{T}+\mathbf{Q}\right)^{-1}$

Moreover, $\mathbf{Q}$ is the covariance matrix of the observation noise, $[\mathbf{H}(k)]_{p}$ is the Jacobian of the observation model, and is defined as follows:

$$
[\mathbf{H}(k)]_{p}=\left[\begin{array}{cc}
-\frac{x_{l / p}(k)}{r_{l / p}(k)} & \frac{y_{l / p}(k)}{r_{l / p}(k)} \\
-\frac{y_{l / p}(k)}{r_{l / p}(k)^{2}} & -\frac{x_{l / p}(k)}{r_{l / p}(k)^{2}}
\end{array}\right]
$$

\subsubsection{Important Weight}

The new particles calculated by the prediction step are weighted to reflect the observation $\mathbf{z}(k)$, and the particles are normalized. The important weight for each particle is defined by the following equation.

$$
[\omega(k)]_{p}=\frac{1}{\sqrt{\left|2 \pi[\mathbf{Q}(k)]_{p}\right|}} \exp \left(-\frac{1}{2}\left(\mathbf{z}(k)-[\hat{\mathbf{z}}(k)]_{p}\right)^{T}[\mathbf{Q}(k)]_{p}\left(\mathbf{z}(k)-[\mathbf{z}(k)]_{p}\right)\right)
$$

with the covariance matrix

$$
[\mathbf{Q}(k)]_{p}=[\mathbf{H}(k)]_{p}\left[\mathbf{\Sigma}_{l}(k-1)\right]_{p}[\mathbf{H}(k)]_{p}{ }^{T}+\mathbf{Q}
$$

\subsubsection{Resampling}

The resampling step has the objectives of restraining the estimation error that occurs when a particle variance increases. In this study, we apply low variance resampling method for the resampling step to restrain an estimation error. The standard resampling step selects independently some particles from temporally particle set by using some random numbers. Meanwhile, the low variance resampling method selects a particle in accordance with probability proportional to the important weight with a single random number. In this way, the low variance resampling method is enable to selects properly a particle than the standard one because it uses a single random number. This random number $r$ is decided from the interval [0 $\left.\mathrm{m}^{-1}\right]$. Therefore, the index $U$ is defined as follows:

$$
U=r+(p-1) m^{-1}
$$

The particle $i$ is selected by the following conditional expression.

$$
i=\underset{p}{\operatorname{argmin}} \sum_{p=1}^{m}[\omega(k)]_{p} \geq U
$$

The new particle set is decided with repeating this process $m$ times. 


\subsection{Detection Method}

The resampling step should not be performed in every step because the variance of the particles is reduced and the estimation error increases. It is necessary that the resampling condition is innovated to reduce the estimation error. Therefore, we apply the variable of the important weight to the resampling condition. A space rover can perform the resampling if the variance of important weight is large. i.e., we propose the effective sample size (ESS) that expresses the variance of important weight. The sample size is defined as the following equation.

$$
\mathrm{ESS}=\left(\sum_{p=1}^{m}[\omega(k)]_{p}^{2}\right)^{-1}
$$

If this is the resampling condition, ESS satisfies the following inequality.

$$
\text { ESS }<\frac{m}{\alpha}
$$

The particles retain the map information of the landmark as shown in (11). In this way, FastSLAM promises the possibility of the robust mapping for a change in terrain because the many particles keep the different map information. However, the different map information that each particle retains converges if a space rover observes the same landmark at same position for a long time. This means that the important weight of each particle occurs the bias notably, and ESS decreases. As a result, the resampling number of times increases by the resampling condition expressed in (23). This characteristics is used to detect local minima in this paper.

\subsection{Escape Method}

The mean $\mu$ of the particles used for estimating the state of a space rover is shifted by $\Delta \mu$ toward the front of a space rover when the rover is steered to the destination to detect local minima. The covariance $\boldsymbol{\Sigma}$ is varied by $\Delta \mathbf{\Sigma}$ according to average relative distance between the landmarks and a space rover. The distribution of the particles, which is represented by the following equation, is spread in the vicinity of the local minima.

$$
f\left(\mathbf{x}_{R}\right)=\frac{1}{2 \pi \sqrt{\left|\mathbf{\Sigma}_{\alpha}\right|}} \exp \left\{-\frac{1}{2}\left(\mathbf{x}_{R}-\mu_{a}\right)^{T} \mathbf{\Sigma}_{\alpha}^{-1}\left(\mathbf{x}_{R}-\mu_{a}\right)\right\}
$$

Here $\boldsymbol{\mu}_{\alpha}=\boldsymbol{\mu}+\Delta \boldsymbol{\mu}$ denotes the mean of the position spread the particles, $\boldsymbol{\Sigma}_{\alpha}=\mathbf{\Sigma}+\Delta \mathbf{\Sigma}$ the covariance of the position, and the subscript $a$ the spread particles. A repulsive potential is generated by using the spread particles that is regarded as repulsive particles. By spreading these repulsive particles towards the front of a space rover, it can escape local minima more smoothly than conventional method.

\section{Experiments}

Fig.4 shows the block diagram of proposed control system. $\mathbf{x}_{d}$ denotes the destination of a space rover, $V_{d}$ and $\theta_{d}$ the velocity command and the heading angle command calculated by the potential function. respectively. $x_{R}, y_{R}$, and $\theta_{R}$ express the position of the $x$ coordinate, the $y$ coordinate, and the attitude angle found by dead reckoning that estimates the state from the angular velocity of wheels in accordance with kinematics, respectively. $V$ is the translational velocity of a space rover. $r_{i}$ and $\varphi_{i}$ are the relative distance and the relative angle between a space rover and landmark observed with sensors mounted on a space rover. $\widehat{x}_{R}, \hat{y}_{R}$ and $\widehat{\theta}_{R}$ are the state estimated with FastSLAM. We carry out the control of a space rover based on these data.

We conduct an experiment to verify the validity of the proposed repulsive particles. Figs. 5 to 7 show the overview of the experimental environment, the system constitution, and the developed space rover, respectively. The infrared sensor is 
used to measure the position of the landmark, and the stepping motor is used as motor. The true trajectory of a space rover is acquired by using a web camera. Fig.8 represents the experimental results when using the proposed repulsive particles. It can be seen from Figs. 8(a) to (d) that a space rover escapes the local minimum and reach the destination smoothly.

\section{Conclusion}

We proposed the novel methods of escaping local minima with the repulsive particles and detecting local minima by the number of times of resampling in FastSLAM. Results of experiment showed that the proposed method was useful for guidance and control of a space rover in unknown environment. We will consider path optimization in future work.

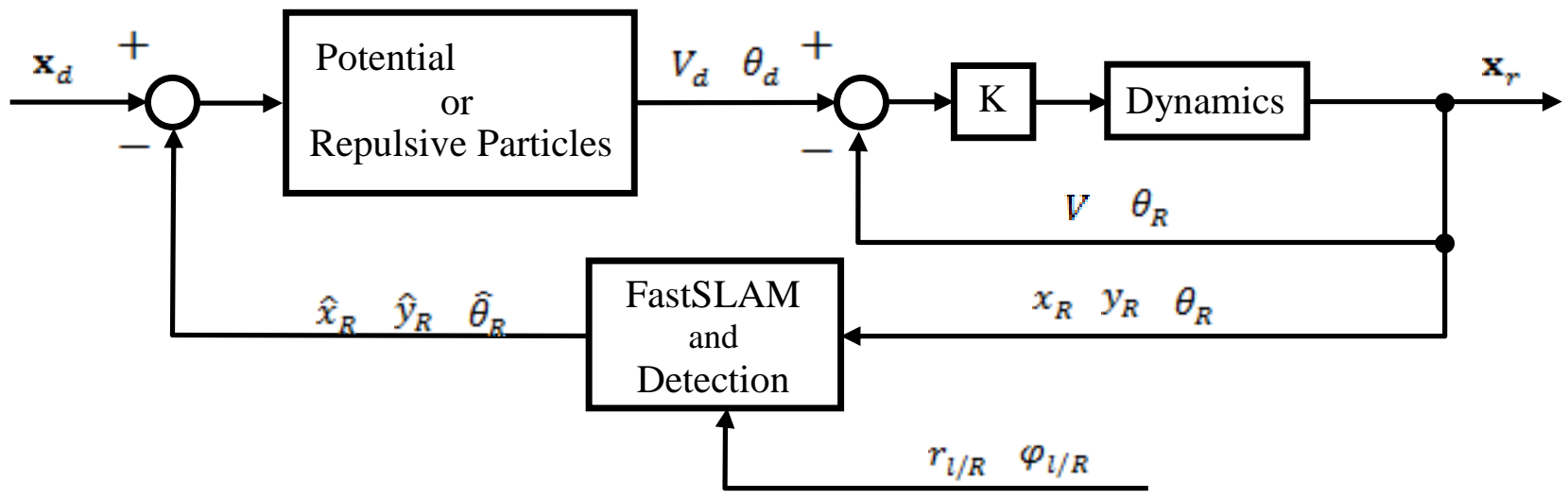

Fig. 4: Block diagram of proposed control system.

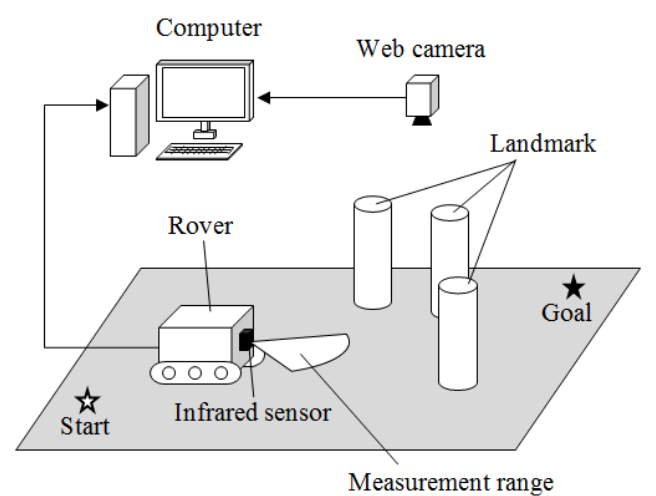

Fig. 5: Overview of experimental environment. 


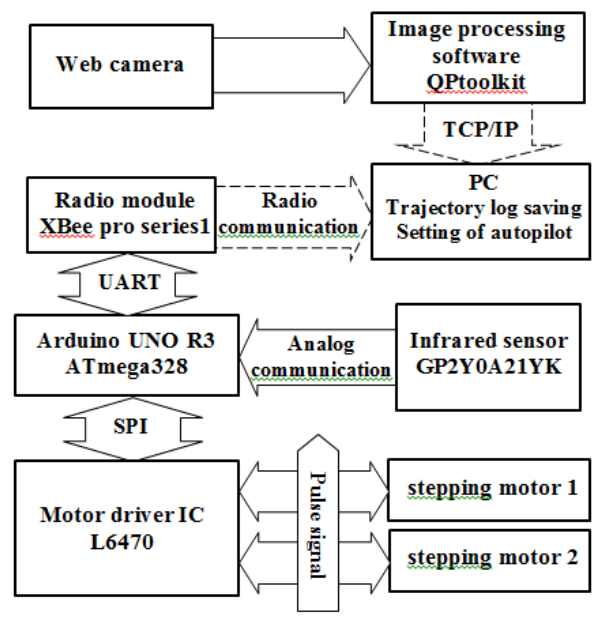

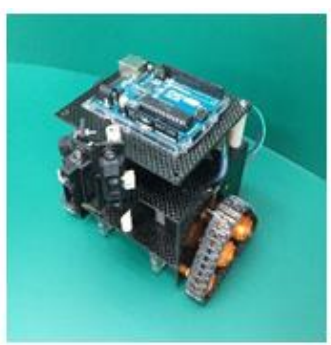

(a) Overall view

Fig. 6: System constitution.

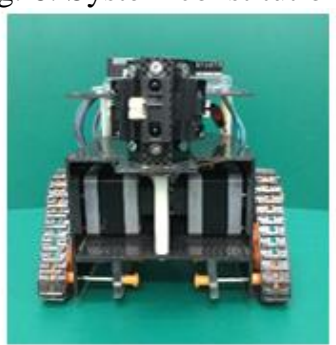

(b)Front

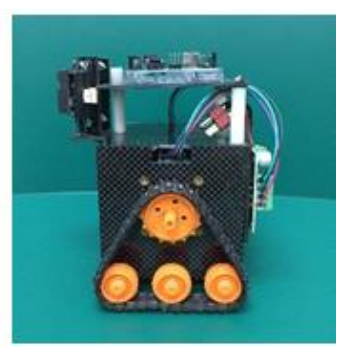

(c) Side

Fig. 7: Overview of space rover.

Table 1. Parameters of FastSLAM

\begin{tabular}{|c|c|}
\hline Number of particles, $M$ & 100 \\
\hline Covariance matrix of predict, $R$ & {$\left[\begin{array}{cc}0.1 & 0 \\
0 & 0.01\end{array}\right]$} \\
\hline Covariance matrix of observation, $Q$ & {$\left[\begin{array}{ccc}0.01 & 0 & 0 \\
0 & 0.01 & 0 \\
0 & 0 & 0.01\end{array}\right]$} \\
\hline Threshold of resampling, $\alpha$ & 2 \\
\hline Mean of repulsive particles, $\mu_{a}$ & {$[2.7,3.0]$} \\
\hline Variance of repulsive particles, $\sigma_{\alpha}$ & 0.1 \\
\hline
\end{tabular}

Table 2. Parameters of potential function.

\begin{tabular}{|c|c|}
\hline$C_{\alpha}$ (attractive gradient) & 0.09 \\
\hline$C_{r}$ (repulsive gradient) & 0.06 \\
\hline$L_{\alpha}$ (sharpness $)$ & 0.3 \\
\hline$L_{r}$ (area of influence $)$ & 0.4 \\
\hline
\end{tabular}




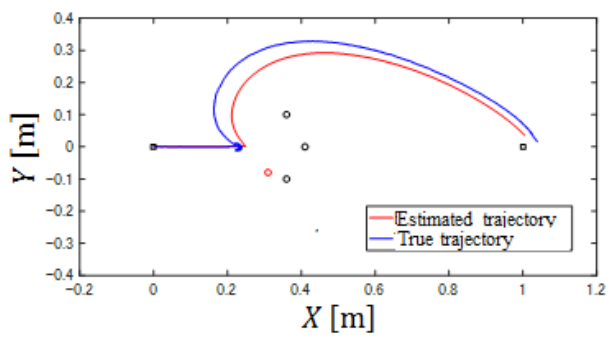

(a) Trajectory

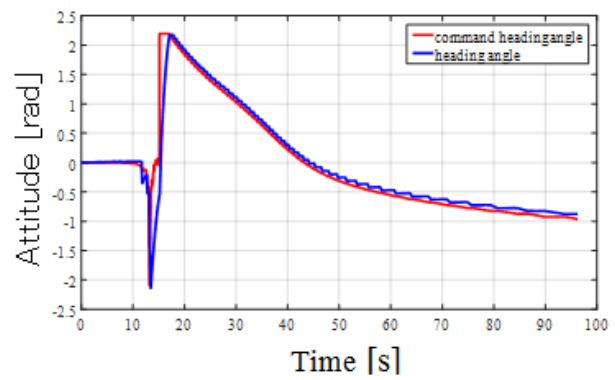

(c) Time histories of heading angle

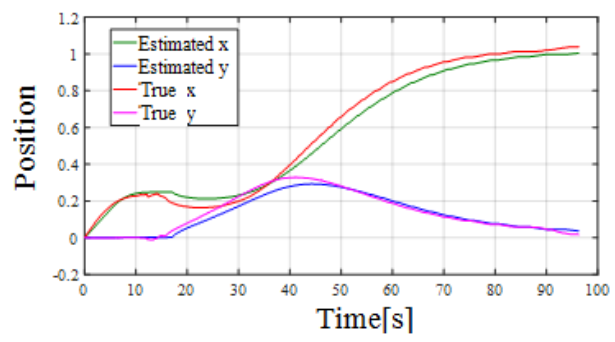

(b) Time histories of position

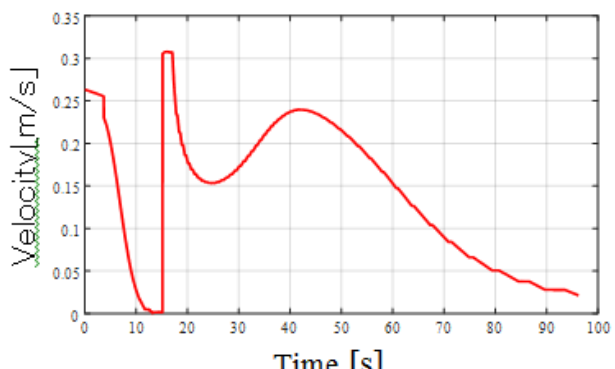

(d) Time history of command velocity

\section{References}

Fig. 8: Experimental results of space rover using proposed repulsive particles.

[1] K. Shibuya, K. Uchiyama, "Potential Field Approach with ATPSA for Planetary Exploration," Proceeding of 58th Space Science and Technology Conference, JSASS-2014-4473, 2014. (in Japanese)

[2] S. Thrun, W. Burgard, D. Fox, "Probabilistic Robotics," MIT press, pp. 96-114, pp. 438-481, 2005.

[3] M. Montemerlo, S. Thrun, D. Koller, B. Wegbreit, "FastSLAM2.0; An Improve Particle Filtering Algorithm for Simultaneous Localization and Mapping That Provably Converges," Proceedings of IJCAI, pp. 1151-1156, 2003.

[4] P. Newman, S. Clark, "A Solution to the Simultaneous Localization and Map Building (SLAM) Problem," Transctions on Robotics and Automation, pp. 229-241, 2002.

[5] K. Nakai, K. Uchiyama, "Vector Fields for UAV Guidance Using Potential Function Method," AIAA Guidance Navigation, and Control Conference, AIAA-2013-4626, 2013.

[6] M. Miki, T. Hiroyasu, M. Kasai, K. Ono, T. Jitta, "Temperature Parallel Simulated Annealing with Adaptive Neighborhood for Continuous Optimization Problem," Proceedings of ISDA, pp.149-154, 2002.

[7] M. Miki, T. Hiroyasu, K. Ono, "Temperature Parallel Simulated Annealing with Adaptive Neighborhood," Information Processing Society of Japan, vol.4 4, no. 4, pp. 745-753, 2001. (in Japanese)

[8] Q. Li, L. J Wang, B. Chen, “An Improved Artificial Potential Field Method for Solving Local Minimum Problem," the $2^{\text {nd }}$ International conference on Intelligent Control and Information Processing, pp. 420-424, 2011. 